\title{
La rentabilidad social en las radios públicas locales. Comparativa de aplicabilidad del indicador IRSCOM en Andalucía y la región de Murcia
}

\author{
Social profitability in local public radio. Applicability comparative of \\ the IRSCOM indicator in Andalucia and the region of Murcia
}

\author{
Dr. José Antonio Sánchez-Hernández, Universidad de Murcia (España) \\ jantoniosanchez@um.es|http://orcid.org/0000-0002-7980-9288 \\ Dr. Manuel Chaparro Escudero, Universidad de Málaga (España) \\ mch@uma.es|http://orcid.org/0000-0002-5630-4009
}

\begin{abstract}
Resumen
Las concesiones de las emisoras de radio públicas como conquista democrática de los gobiernos locales, reguladas por Ley desde 1991, obedecen a una decidida apuesta por vertebrar territorios, aportar cohesión social, participación, valores y potenciar la identidad cultural. Este artículo trata de establecer las claves de la rentabilidad social de las radios municipales para mejorar la prestación de un servicio esencial.

La investigación también trata de conocer cómo se distribuyen y articulan a lo largo del ámbito autonómico de Murcia estas emisoras y replicar la herramienta IRSCOM (índice de Rentabilidad Social en Comunicación) desarrollada por el Laboratorio de Comunicación y Cultura COMandalucía de la Universidad de Málaga, dentro de los proyectos I+D+i "Plan Nacional I+D+i. CSO2011-29195 y Proyecto de Investigación de Excelencia. P10 TIC 6593 (Junta de Andalucía): "La radio y la televisión local en Andalucía: situación actual e incidencia social”, para determinar si se cubren las necesidades comunicativas para las que están concebidas.

La experiencia investigativa parte de la aplicación del indicador IRSCOM a más cien emisoras en Andalucía. Esta experiencia de réplica nos ha permitido conocer la situación de la Comunidad Autónoma de la Región de Murcia (CARM) y hacer un balance comparativo con las emisoras analizadas.
\end{abstract}


Los resultados buscan poner de manifiesto las fortalezas y debilidades de estos medios para proponer medidas de intervención. En este sentido se persigue comprobar si el indicador, IRSCOM, es una herramienta de análisis útil para ser aplicada a otros contextos de análisis a los medios de proximidad públicos.

\section{Abstract}

The public radio station concessions as a democratic conquer of the local governments, ruled by the Law since 1991, give answer to a firm decision so as to vertebrate territories, give social cohesion, participation, values and to promote the cultural identity. This article aims to establish the local radio social rentability keys for improving the provision of an essential service.

This investigation also aims to know how radio stations are distributed and articulated along the autonomous Region of Murcia and to replicate the IRSCOM tool (Social Rentability in Communication Index) developed for the Communication and Culture Laboratory COMandalucia from the University of Malaga, within both the I+D+l "I+D+l. CSO2011-29195 National Plan and the Excellence Project of Investigation. P10 TIC 6593 (Junta of Andalusia): "The radio and local television in Andalusia: current situation and social impact", so as to determine whether the communicative needs are covered for what they are conceived.

This investigative experience starts from the application of the IRSCOM indicator in more than a hundred of radio stations in Andalusia. This experience of replica has gave us the opportunity to understand the situation of the Autonomous Community of the Region of Murcia (CARM) and to establish a comparison among the stations that have been analysed.

Palabras clave: Emisoras municipales, radio pública local, rentabilidad social, políticas públicas, IRSCOM.

Keywords: Local radio stations, local public radio, social profitability, public politics, IRSCOM.

\section{INTRODUCCIÓN}

La transformación del ecosistema mediático impulsado por las TIC ha supuesto una profunda modificación de los diferentes paradigmas de los medios de comunicación tradicionales y la aparición de nuevos medios. Sin duda, el principal motor de este proceso ha sido la convergencia con Internet y con la telefonía móvil. La radio, que de manera histórica se ha adaptado perfectamente a este tipo de disrupciones, ha aprovechado los nuevos entornos comunicativos generados para evolucionar y romper con sus limitaciones tradicionales (cobertura, recursos expresivos, interactividad,...). 
Estos nuevos entornos comunicativos convergen con plataformas como Facebook, Twitter, iVoox, etc., propiciando nuevas interacciones desde la proximidad, nuevas dinámicas en la participación de la ciudadanía. En definitiva, un periodismo ciudadano que pone el acento en la realidad cercana y de afectación directa, capaz de admitir la información enviada y hasta producida por el común. La narrativa de instantaneidades y atemporalidad que propicia los nuevos usos tecnológicos no altera la narratividad de lo cotidiano y la proximidad, los contenidos producidos deben obedecer a una especialización territorial que continúa siendo la principal seña de identidad. En este sentido, los medios públicos locales refuerzan su apuesta por los contenidos locales, al tiempo que deben aprender, a través de la elaboración de nuevos mapas temáticos y fuentes, a incidir desde la proximidad en las transiciones globales ante los desafíos de nuestro modelo de vida, ¿cómo intervenir ante la crisis climática, ética, económica que repercute en lo cotidiano? Los mapas temáticos permiten fijar los asuntos de interés para la ciudadanía desde una perspectiva superadora de la disyuntiva global-local, para buscar respuestas locales de intervención de incidencia global desde la comunicación de modelos de buenas prácticas. Esa debe ser la auténtica razón que justifica la existencia de los medios de proximidad pública y asociativos como constructores de tejido ciudadano desde proyectos sin ánimo de lucro.

La participación ciudadana y el compromiso municipal por proporcionar a sus emisoras los recursos necesarios para desarrollar con las garantías de calidad necesarias este servicio público municipal desde la independencia, pluralidad y transparencia constituyen los dos ejes fundamentales que determinan las dinámicas de estos medios de proximidad y sobre los que se fundamenta esta investigación.

Una adecuada apuesta municipal por dotar a las emisoras de radio de los recursos humanos y materiales necesarios para una correcta prestación de este servicio actúa como un elemento garante del desarrollo de un proyecto comunicativo basado en principios de servicio público, evitando una inoportuna deriva comercial que se produce cuando el medio tiene una excesiva dependencia de los recursos procedentes de la comercialización publicitaria. En este sentido: la mercantilización de la información y la comunicación, de las redes de distribución, de las tecnologías de la información, ha provocado el desmantelamiento y empobrecimiento de los medios públicos. El concepto de servicio público ya no es aplicable a los medios comerciales, lo que supone una cierta privatización del espectro radioeléctrico y un nulo compromiso por los contenidos de utilidad para la ciudadanía.

Por este motivo, las emisoras municipales de radio se conciben como un servicio público que contribuye a la vertebración del territorio, facilitando la cohesión y el sentimiento identitario 
de sus habitantes. Los ciudadanos sienten suya la emisora cuando intervienen en la toma de decisiones mediante consejos de participación ciudadana, cuando se implican en la programación de la emisora generando contenidos, cuando participan en cursos y talleres organizados por el medio, cuando utilizan sus fondos bibliográficos, fonográficos e instalaciones, etc. Estos medios se fundamentan en un necesario modelo de comunicación participativa que propicia el conocimiento de la realidad más próxima mediante la articulación de la intervención ciudadana. De esta manera, se convierte en el "medio idóneo para movilizar y dar espacios a los ciudadanos donde puedan crear imaginarios propios y no impuestos" (Gabilondo, 2015, p. 298).

\section{OBJETIVOS}

El objeto principal de estudio consiste en determinar la rentabilidad social de las emisoras municipales de radio murcianas que se encuentran operativas y que son gestionadas directamente por los respectivos ayuntamientos concesionarios del servicio. Por tanto, quedan excluidas aquellas emisoras cuya gestión y/o programación han sido externalizadas en fraude de ley y se prestan por terceros que no mantienen una relación laboral directa con el ente municipal.

Esta labor de identificación y cribado inicial permitirá diseñar el mapa de las emisoras municipales de radio de la CARM, reflejando aquellos municipios que poseen licencia, las emisoras operativas, las externalizadas, las que han sido cerradas y, finalmente, las que no se han puesto aún en servicio.

Nuestra hipótesis de partida es que las emisoras municipales de radio murcianas no han desarrollado todo su potencial comunicativo para alcanzar unos niveles aceptables en lo que al indicador de rentabilidad social se refiere.

\section{METODOLOGÍA}

Como punto de partida, establecemos las características principales de esta realidad comunicativa para, seguidamente, fijar la distribución territorial de las emisoras municipales de radio en la CARM, y finalmente, determinar su rentabilidad social a partir del IRSCOM. En este sentido, relacionaremos los resultados obtenidos con las investigaciones sobre esta misma realidad comunicativa realizadas en la Comunidad Autónoma de Andalucía y publicadas entre 2014 y $2015^{1}$.

La investigación se ha desarrollado entre los meses de septiembre y diciembre de 2017 mediante la realización de una encuesta presencial a los responsables de las diferentes emisoras municipales de radio murcianas, complementada con una observación directa de la realidad 
comunicativa de los medios estudiados, visitando las propias dependencias y analizando las características de sus portales comunicativos y de sus emisiones.

\section{POLÍTICAS PÚBLICAS DE COMUNICACIÓN Y EMISORAS MUNICIPALES DE RADIO}

Hasta el último cuarto del siglo XX, la escasa implantación de medios de comunicación locales y el poco interés de los medios regionales por los contenidos de proximidad de los núcleos urbanos rurales, han propiciado una escasa cobertura informativa de estos entornos de los que, en ocasiones, solo se recogían aquellas informaciones especialmente sensibles o graves (sucesos) o por criterios de oportunidad política (inauguraciones, proyectos).

Es a finales de la década de los setenta cuando la comunicación local comienza a ser puesta en valor con las primeras emisoras municipales de radio, gracias a la llegada de la democracia, a las nuevas posibilidades tecnológicas que permitieron un abaratamiento de los equipos emisores y a un cierto afán de protagonismo y notoriedad ante los micrófonos (Chaparro, 1998).

Fueron los movimientos sociales de esta época, que reclamaban mayor libertad de expresión, y la iniciativa de las primeras alcaldías democráticas, las circunstancias que facilitaron la apertura del espacio radioeléctrico para el ejercicio del derecho de comunicación. Cataluña, Andalucía y posteriormente, Galicia fueron las comunidades más activas y aún hoy siguen siendo referentes (Gabilondo, 2014 y Chaparro, 1998).

La Constitución Española de 1978 propició la construcción del Estado de las autonomías permitiendo desarrollar "políticas más eficientes encaminadas al equilibrio y la vertebración territorial [...] pero, en el caso que nos ocupa, la ausencia de políticas públicas [efectivas] de comunicación, hasta la fecha, ha marcado una realidad que se ha construido a sí misma" (Gabilondo, 2014, p. 165).

En el desarrollo normativo cobran especial relevancia dos disposiciones. La primera de ellas es la Ley 7/1985, de 2 de abril, Reguladora de las Bases del Régimen Local, que garantiza el uso de los medios públicos por parte "de las asociaciones para la defensa de los intereses generales o sectoriales de los vecinos" (art. 72). La segunda es la Ley 11/1991, de 8 de abril, de Organización y Control de las Emisoras Municipales de Radiodifusión Sonora en Frecuencia Modulada (LOCEM). Esta última disposición fue derogada por la Ley 7/2010, de 31 de marzo, General de Comunicación Audiovisual que contempla la posibilidad de la privatización del servicio, previa regulación por la Comunidad Autónoma competente. Esta circunstancia que viene a desvirtuar los principios inspiradores de estos medios públicos participativos y alejados de fines mercantilistas, no se ha producido todavía en ninguna Comunidad, aunque como veremos algunas alcaldías han optado unilateralmente por situarse fuera de la Ley externalizando el servicio. 
Dentro de este nuevo marco regulatorio se sitúa la Ley 10/2018, de 9 de octubre, Audiovisual de Andalucía, que establece que este servicio público audiovisual "podrá ser prestado, siempre bajo el régimen de gestión directa, por la Administración de la Junta de Andalucía, las entidades locales de Andalucía, las universidades públicas y los centros docentes públicos no universitarios" (art. 44.1); aunque podrá contar con la colaboración de otras entidades y personas cuando sea necesaria la disponibilidad de medios materiales o profesionales ajenos al ente o a la sociedad responsable de la gestión directa del servicio... [excepto para] contenidos de difusión de información diaria, ni podrá conllevar merma de capacidad productiva, ni de recursos humanos o técnicos públicos, ni de la calidad de los servicios que se prestan (art. 46.4).

Por su parte, la CARM adquiere las competencias legislativas y ejecutivas en materia de prensa, radio, televisión y otros medios de comunicación social, en virtud de la Ley Orgánica 9/1992, de 23 de diciembre, de transferencia de competencias a Comunidades Autónomas que accedieron a la autonomía por la vía del artículo 143 de la Constitución. Las comunidades reconocidas como históricas (Andalucía, Cataluña, Galicia y País Vasco) tenían este derecho transferido desde 1983.

Entre 1994 y 2005, la CARM otorgó las concesiones a los ayuntamientos solicitantes de emisoras municipales de radio, sin que hasta la fecha se hayan producido nuevas solicitudes de licencias.

\section{EL MAPA DE LAS EMISORAS MUNICIPALES DE RADIO EN LA CARM}

En la CARM existen 45 ayuntamientos, de ellos 21 (47\%) poseen licencia para gestionar una EM. Sin embargo, no todas las licencias concedidas se encuentran operativas ni se gestionan de acuerdo con lo establecido en la normativa específica.

Estos factores permiten distinguir las diferentes situaciones en las que se encuentran actualmente estos medios y que, además, posibilitan realizar el mapa radiofónico público local siguiendo la clasificación propuesta por COMandalucía (Figura 1):

a) Emisoras municipales operativas: aquellas que actualmente emiten de manera regular y que cumplen con la normativa por la que se les ha otorgado la concesión administrativa. En esta situación se encuentran 12 de las 21 licencias concedidas (57\%).

b) Emisoras municipales externalizadas: casos en que los ayuntamientos gestionan este servicio de manera indirecta mediante la cesión a terceros de la gestión. La Ley 7/2010, de 31 de marzo, General de Comunicación Audiovisual (LGCA), contempla en su artículo 40.2 que "las Comunidades Autónomas podrán acordar transformar la gestión directa del servicio en gestión indirecta, mediante la enajenación de la titularidad de la entidad 
prestadora del servicio". En el informe elaborado por el Laboratorio de Comunicación y Cultura COMandalucía de la Universidad de Málaga (mayo de 2017) y la Asociación para la Investigación de Medios (iMEDEA) ${ }^{2}$, se advierte que los ayuntamientos no tienen competencias para modificar las condiciones de gestión recogidas en la concesión de licencia que en su día le fue otorgada por la comunidad autónoma correspondiente, en base a la derogada Ley 11/1991, de 8 de abril, de Organización y Control de las Emisoras Municipales de Radiodifusión Sonora en Frecuencia Modulada (LOCEM). Al no existir un desarrollo legislativo de dicha materia por parte de la CARM, los ayuntamientos no tienen competencias para modificar el régimen jurídico de la concesión obtenida al amparo de la LOCEM que solo contemplaba la gestión directa de este servicio. En el caso de Murcia tres emisoras se gestionan mediante contrato administrativo de prestación de servicios con terceros sin ninguna relación laboral directa con los respectivos ayuntamientos, que en esta situación cometerían un delito de prevaricación y malversación.

c) Existen cinco emisoras públicas locales cerradas: aquellas que, tras un periodo de emisión, al no existir una apuesta municipal consistente o por circunstancias sobrevenidas han dejado de emitir.

d) Emisoras municipales con licencia sin activar: concesiones otorgadas por la CARM y que no han llegado a ponerse en funcionamiento en ningún momento. El único caso es el del municipio de Totana (107.0 FM).

\section{Figura 1. Mapa de las emisoras municipales de radio murcianas}

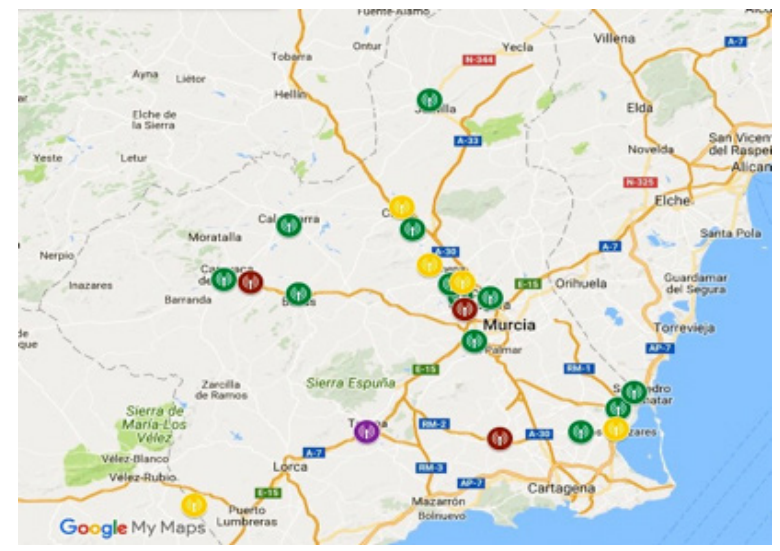

Operativas (verde), externalizadas (rojo), cerradas (amarillo) y sin activar (púrpura)

Fuente: Investigación propia (2017)

En la Figura 1 podemos observar como la CARM dispone, actualmente, de doce emisoras municipales de radio operativas con gestión no externalizada; tres emisoras se encuentran operativas pero externalizadas; cinco emisoras están cerradas; y finalmente, una licencia no ha sido activada. 
Atendiendo a cifras de población, las emisoras municipales de radio murcianas se ubican en municipios entre 9.000 y 70.000 habitantes. Los tres municipios con más de 70.000 habitantes no disponen de este servicio ya que son territorios donde existe un mayor número de emisoras comerciales, la mayoría pertenecientes a cadenas de radio que efectúan desconexiones puntuales para ofrecer la programación local. Tampoco cuentan con emisora municipal las trece poblaciones con menos de 9.000 habitantes, debilidades no achacables a temas presupuestarios si se considera que son servicios esenciales. De los veinte municipios con poblaciones comprendidas entre 9.000 y 26.000 habitantes once disponen de EM, sumando el $73 \%$ de las quince existentes. Las cuatro restantes se ubican en poblaciones que cuentan entre 26.000 y 70.000 habitantes. Así pues, la radio pública local murciana es mayoritaria en las poblaciones entre 9.000 y 26.000 habitantes, situación similar a la analizada en la Comunidad Autónoma de Andalucía (Gabilondo, 2014). Los mayores recursos y la necesidad de cobertura informativa de contenidos de proximidad no satisfecha por otros medios son los principales incentivos (Sánchez-Hernández, 2012). (Tabla 1).

Resulta necesario articular iniciativas para conseguir la reapertura de las emisoras de aquellos ayuntamientos que han prescindido del servicio e incentivar el desarrollo de estos proyectos en otras localidades que no lo han solicitado, con el objetivo de revitalizar la comunicación municipal. Se evidencian, de esta manera: las dificultades de llevar a cabo proyectos de comunicación local independientemente de las voluntades políticas e institucionales comprometidas y se confirma la necesidad de incorporar formación especializada, técnica y de dinamización social en gestión y liderazgo, como base de partida para llevar a cabo con éxito este tipo de proyectos. (Gabilondo, 2014, p. 167). 


\begin{tabular}{|c|c|c|c|}
\hline Municipio & Emisora & Población & Situación \\
\hline Molina de Segura & Radio Compañía & 69.614 & Operativa \\
\hline Alcantarilla & Radio Sintonía & 41.155 & Operativa \\
\hline Torre Pacheco & Radio Torre Pacheco & 34.630 & Operativa \\
\hline San Javier & Radio San Javier & 31.782 & Operativa \\
\hline Totana & - & 30.981 & No activada \\
\hline Caravaca de la Cruz & Caravaca Radio & 25.591 & Operativa \\
\hline Jumilla & Radio Jumilla & 25.362 & Operativa \\
\hline San Pedro del Pinatar & Radio Pinatar & 24.660 & Operativa \\
\hline Las Torres de Cotillas & $\begin{array}{c}\text { Onda } 92 \text { - Radio } \\
\text { Cotillas }\end{array}$ & 21.341 & Externalizada \\
\hline Fuente Álamo & Antena Ciudad & 16.205 & Externalizada \\
\hline Cehegín & Más Fm Begastri & 15.321 & Externalizada \\
\hline Abarán & Radio Abarán & 13.183 & Operativa \\
\hline Bullas & La Onda de Bullas & 11.714 & Operativa \\
\hline Ceutí & Onda Color & 11.321 & Operativa \\
\hline Calasparra & Radio Calasparra & 10.268 & Operativa \\
\hline Alguazas & Alguazas Radio & 9.613 & Operativa \\
\hline
\end{tabular}

Fuente: Población a 1-1-2016 (INE). Elaboración propia (2017)

\section{LA RENTABILIDAD SOCIAL DE LAS EMISORAS PÚBLICAS DE PROXIMIDAD EN LA}

\section{CARM}

La razón de ser de las emisoras municipales de radio se fundamenta en ser referencia de la noticia local y facilitar la participación ciudadana. Las EM son un servicio público que se ocupa de los contenidos informativos de su entorno más próximo y que no tienen cabida en otros medios de ámbito provincial, autonómico o nacional. Además, la programación no se sustenta de manera prioritaria sobre aquellos contenidos capaces de seducir a la mayor cantidad de audiencia posible, sino que la emisora acude también al rescate de las historias e informaciones que recogen la actividad de una amplia minoría. 
Por este motivo, nos encontramos ante una realidad comunicativa que debe disponer de los recursos humanos y materiales necesarios para garantizar que se desempeña con las máximas garantías posibles de calidad, independencia, transparencia, pluralidad y participación ciudadana.

El Índice de Rentabilidad Social IRSCOM, nos permite analizar la gestión, la organización y el funcionamiento del medio; la consideración identitaria como servicio público municipal; las características de una programación basada en principios de pluralidad, participación y fomento de la información local; así como su capacidad para interactuar y relacionarse con el resto de actores del sector y colectivos sociales.

IRSCOM es una herramienta específicamente diseñada para determinar la rentabilidad social de los medios audiovisuales mediante criterios cuantitativos y cualitativos ponderables, en nuestro caso aplicado a las EM, como vertebradoras sociales gracias a su capacidad para movilizar la participación de colectivos y colaboradores voluntarios.

El indicador se estructura a partir de la identificación de seis categorías macro: gestión, capital social, articulación territorial, programación, entorno comunicativo e infraestructuras. Estas categorías macro se determinan mediante la evaluación de una serie de áreas de análisis, como son la transparencia, la capacidad dinamizadora, la solvencia relacional del medio dentro del sector, la calidad de la programación, la adaptación al nuevo entorno comunicativo radiofónico y el compromiso de la institución pública en materia de infraestructuras (Tabla 2)3.

\section{Tabla 2. Categorías macro del IRSCOM}

\begin{tabular}{|c|c|c|}
\hline & Categorías macro del IRSCOM & Aspectos que permite la evaluación \\
\hline 1 & Gestión & Transparencia del medio como ente público \\
\hline 2 & Capital social & $\begin{array}{l}\text { Capacidad dinamizadora, construcción de ciudadanía } \\
\text { y potenciación de los recursos humanos }\end{array}$ \\
\hline 3 & Articulación territorial & $\begin{array}{l}\text { Solvencia relacional con asociaciones del sector } \\
\text { audiovisual }\end{array}$ \\
\hline 4 & Programación & $\begin{array}{l}\text { Asunción del medio como servicio público, aplicando } \\
\text { principios de diversidad cultural, conciencia } \\
\text { medioambiental, interés en lo local y derechos } \\
\text { humanos }\end{array}$ \\
\hline 5 & Entorno comunicativo & $\begin{array}{l}\text { Capacidad de adaptación a las nuevas tecnologías, } \\
\text { favoreciendo una mayor difusión del servicio públicoW }\end{array}$ \\
\hline 6 & Infraestructuras & $\begin{array}{l}\text { Compromiso público para unas condiciones dignas } \\
\text { que faciliten el ejercicio de la profesión }\end{array}$ \\
\hline
\end{tabular}

Fuente: COMandalucía (2014) 
Para la interpretación de los valores numéricos obtenidos se establecen intervalos que permiten ponderar cada categoría analizada en función de casuísticas como el tamaño poblacional del núcleo que atiende la emisora, dotación presupuestaria, personas contratadas y colaboradoras. En este sentido, los valores se consideran adecuados cuando la emisora se sitúa en un intervalo que va de un mínimo de 74 a un máximo de 120 puntos (Tabla 3). En este sentido, el mínimo señala el umbral básico para poder desempeñar el trabajo con garantías, y el máximo responde a la situación ideal que le correspondería caso de corregir las deficiencias detectadas (Chaparro, Olmedo y Gabilondo, 2016).

\section{Tabla 3. Puntuaciones de las categorías del IRSCOM}

\begin{tabular}{|l|l|c|c|}
\hline & Categorías & Puntuación mínima & Puntuación máxima \\
\hline 1 & Gestión & 20 & 30 \\
\hline 2 & Capital social & 10 & 21 \\
\hline 3 & Articulación territorial & 6 & 10 \\
\hline 4 & Programación & 26 & 40 \\
\hline 5 & Entorno comunicativo & 5 & 7 \\
\hline 6 & Infraestructuras & 7 & 12 \\
\hline Total de puntos & 74 & 120 \\
\hline
\end{tabular}

Fuente: COMandalucía (2014)

Los valores que alcanzan cada una de las categorías analizadas, colocados en un entorno gráfico, permiten detectar con mayor claridad las debilidades y fortalezas de las emisoras municipales. Por ello: el IRSCOM se ha diseñado con un criterio constructivo, con la finalidad de orientar aquellos aspectos o procesos en los que el medio debe mejorar para obtener mayor impacto social desde un criterio de responsabilidad con sus fines. El logro del indicador es hacer tangible lo intangible en las mejoras de las dinámicas comunicativas que proponen los medios. (Chaparro, Olmedo y Gabilondo, 2016, pp. 55-56).

\subsection{Gestión}

En esta categoría se evalúa el compromiso con los principios de transparencia y acceso a la información pública, el cumplimiento de los estatutos o el reglamento de funcionamiento del servicio, la publicación de la memoria anual en alguna plataforma de fácil acceso a la ciudadanía, 
la existencia de un consejo asesor u órgano de participación vinculado a la sociedad civil, y finalmente, disponer de un enlace desde la página web del ayuntamiento (Chaparro, Olmedo y Gabilondo, 2015).

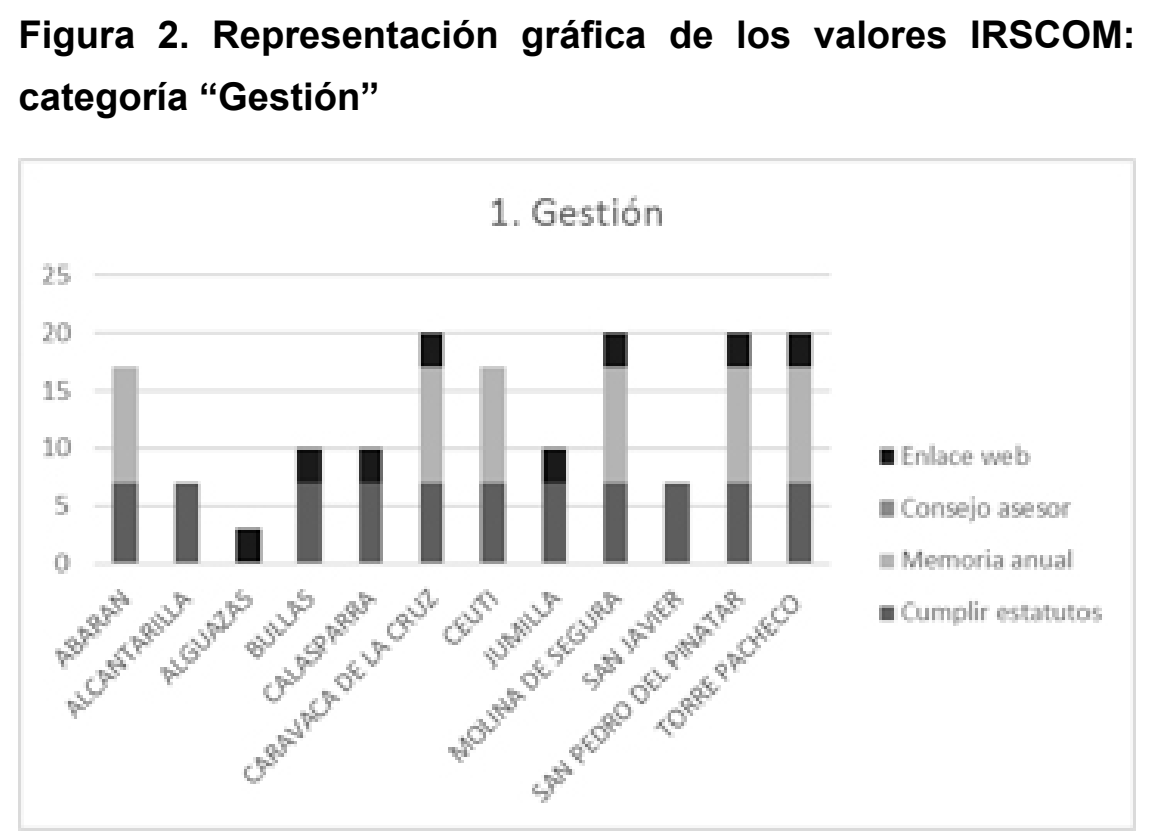

Fuente: elaboración propia (2017)

Las emisoras que se gestionan mediante una sociedad mercantil con capital social exclusivamente público (art. 85.2.d de la Ley 7/1985, de 2 de abril, Reguladora de las Bases del Régimen Local), tienen la obligación de redactar una memoria de gestión anual para su aprobación en el Consejo de Administración y depositar las cuentas en el Registro Mercantil correspondiente. Además, el presupuesto anual de estas sociedades públicas aparece publicado en el Boletín Oficial de la Región de Murcia (B.O.R.M.), incluido y desglosado dentro del presupuesto municipal. En la Figura 2 observamos que las cuatro emisoras que se administran mediante este sistema alcanzan mejores valores en la gestión que el resto. El 92\% de las emisoras (11 de 12) aseguran cumplir los estatutos, aunque solo seis elaboran una memoria anual, ninguna de ellas cuenta con consejo asesor municipal y el 67\% (8 de 12) disponen de un enlace en la web del ayuntamiento.

En Andalucía, de las 112 E.M. analizadas, 76 (68\%) aseguran cumplir los estatutos, lo que se contradice con aquellas que publican la memoria (4 emisoras, 3,5\%). Solo 9 emisoras (8\%) cuentan con consejo asesor y 87 (78\%) disponen de un enlace en la web del ayuntamiento. Los valores mínimos de este campo son superados solo por 4 de las 112 emisoras (Gabilondo, 2015). 
De lo que se desprende que la percepción que tienen las emisoras sobre el cumplimiento de lo establecido en sus estatutos es más elevada en la CARM que, además, publican de manera accesible sus memorias de gestión/económicas anuales, sobre todo aquellas emisoras que se gestionan mediante una sociedad mercantil local con capital municipal. *

\subsection{Capital Social}

Los trabajadores que prestan sus servicios en las diferentes emisoras constituyen uno de sus activos más importantes. En este sentido, en esta categoría evaluamos el número de trabajadores con los que cuenta el medio, el número de mujeres contratadas y de discapacitados como factor de inclusión. La puntuación de estos aspectos se ha ponderado en función de la población de cada municipio con el fin de que reflejen de una manera más objetiva el esfuerzo realizado por ayuntamientos con distinta capacidad presupuestaria. Además, se toma en consideración la participación voluntaria y altruista de colaboradores y de colectivos de la localidad que contribuyen a la democratización y a la apertura social del medio. Finalmente, se aprecia el esfuerzo de la emisora por realizar talleres formativos que faciliten a los ciudadanos desarrollar habilidades comunicativas.

En este ámbito comunicativo adquiere especial importancia que las emisoras municipales de radio cuenten, además, con la implicación de colabores individuales y colectivos ya que constituyen "una participación indispensable en la difusión de nuevas realidades, demandas e ideas emprendedoras que fomenten la integración de los habitantes y de las poblaciones, muchas de ellas en proceso de cambio social y demográfico como consecuencia de los flujos migratorios" (Gabilondo, 2015, p. 260).

Ninguna de las emisoras municipales murcianas se sitúa en el umbral mínimo necesario de esta categoría (10 puntos, de un máximo de 21). En este campo, se aprecia un déficit en la dotación de personal, una razonable estructuración de la plantilla en función del género, la ausencia de trabajadores con discapacidad y una importante presencia de colaboradores y colectivos como se aprecia en la Figura 3.

La situación se encuentra más armonizada en Andalucía, donde cuentan con 53 E.M. (47\%) que se encuentran en un nivel óptimo y 59 (53\%) que no superan la puntuación mínima requerida. En esta comunidad autónoma, en poblaciones menores de 10.000 habitantes se han contabilizado 73 contratados (18\% del total), 421 colaboradores (39\%) y 186 colectivos (37\%). La situación es parecida en las poblaciones entre 10.000 y 20.000 habitantes, no es tan alta como podría esperarse en las de más de 20.000 y destaca el número de contratados en las poblaciones de 
más de 50.000 habitantes, dato sobredimensionado por las altas concentraciones en emisoras como Jerez (87) y Onda Azul Málaga (34) (Gabilondo, 2014).

\section{Figura 3. Representación gráfica de los valores IRSCOM: categoría} "Capital social"

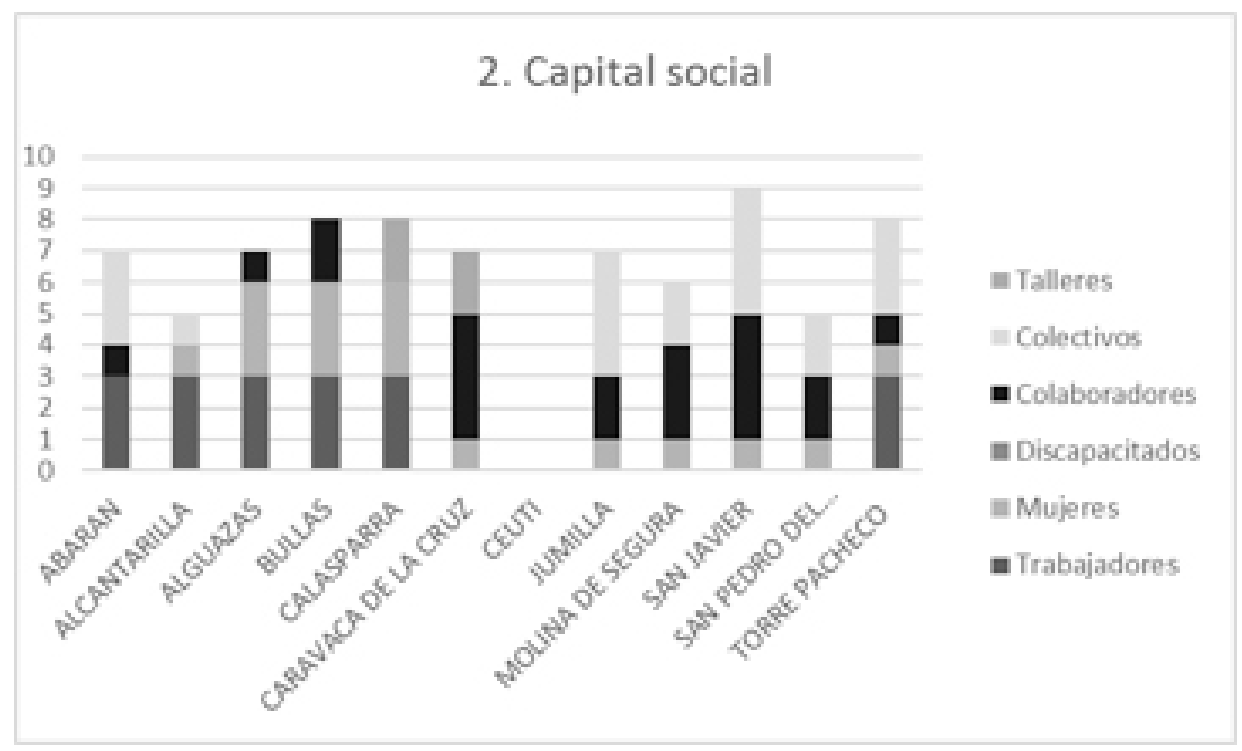

Fuente: elaboración propia (2017)

En la CARM, los datos reflejan un notable déficit en el número de trabajadores que integran las plantillas de las diferentes EM en función de la población de los municipios. Solo cuenta con una emisora en una población de menos de 10.000 habitantes que dispone de un trabajador (3\% del total) y dos colaboradores (3\% del total). Las cuatro emisoras de municipios entre 10.000 y 20.000 habitantes tienen siete trabajadores $(23 \%)$, nueve colaboradores $(11 \%)$ y cuatro colectivos $(12 \%)$. Son los siete municipios con poblaciones de más de 20.000 habitantes los que suman más trabajadores, $23(74 \%)$, Igual ocurre con los colaboradores y colectivos, 72 (86\%) y 32 (88\%), respectivamente. (Tabla 4$)$. 
Tabla 4. Distribución de trabajadores, colaboradores y colectivos en las diferentes emisoras

\begin{tabular}{|l|c|c|c|c|c|}
\hline \multicolumn{1}{|c|}{ Municipio } & Emisora & Población & $\begin{array}{c}\text { Trabajado- } \\
\text { res }\end{array}$ & $\begin{array}{c}\text { Colaborado- } \\
\text { res }\end{array}$ & Colectivos \\
\hline $\begin{array}{l}\text { Molina de } \\
\text { Segura }\end{array}$ & Radio Compañía & 69.614 & 4 & 12 & 2 \\
\hline Alcantarilla & Radio Sintonía & 41.155 & 5 & 0 & 1 \\
\hline Torre Pacheco & $\begin{array}{c}\text { Radio Torre } \\
\text { Pacheco }\end{array}$ & 34.630 & 4 & 2 & 5 \\
\hline San Javier & Radio San Javier & 31.782 & 3 & 25 & 12 \\
\hline $\begin{array}{l}\text { Caravaca de } \\
\text { la Cruz }\end{array}$ & Caravaca Radio & 25.591 & 3 & 19 & 0 \\
\hline Jumilla & Radio Jumilla & 25.362 & 1 & 6 & 10 \\
\hline $\begin{array}{l}\text { San Pedro del } \\
\text { Pinatar }\end{array}$ & Radio Pinatar & 24.660 & 3 & 8 & 2 \\
\hline Abarán & Radio Abarán & 13.183 & 2 & 2 & 4 \\
\hline Bullas & La Onda de Bullas & 11.714 & 2 & 7 & 0 \\
\hline Ceutí & Onda Color & 11.321 & 1 & 0 & 0 \\
\hline Calasparra & Radio Calasparra & 10.268 & 2 & 0 & 0 \\
\hline Alguazas & Alguazas Radio & 9.613 & 1 & 2 & 0 \\
\hline
\end{tabular}

Fuente: elaboración propia (2017)

De los 31 trabajadores, catorce son mujeres (45\%) con lo que casi se alcanzan criterios de equidistribución en función del género. Se aprecia en general, una importante presencia de colaboradores (83) y colectivos (36) en la programación de estos medios.

Comparativamente, la existencia en Andalucía de un mayor tejido radiofónico municipal en poblaciones de menos de 10.000 habitantes proporciona una importante plataforma comunicativa para estas localidades donde se genera un importante porcentaje de empleo y unas considerables cuotas de participación ciudadana.

La elevada presencia de colaboradores y colectivos pone de manifiesto la configuración de estas emisoras como medios de comunicación participativos, donde los ciudadanos y colectivos sociales intervienen en la construcción de su propio imaginario colectivo, pero para ello los recursos humanos deben contribuir a la gestión de este servicio público municipal. La emisora 
tiene que desarrollar una serie de tareas fundamentales: producir la programación y los anuncios, garantizar la continuidad de las emisiones y la administración, acometer el mantenimiento de equipos e instalaciones, etc. En la mayoría de los casos se observa que los trabajadores atesoran una notable polivalencia funcional y soportan una elevada saturación de tareas.

\subsection{Articulación territorial}

En esta categoría se evalúa la pertenencia a redes de emisoras y su aportación de producción propia para ser compartidos con la misma. La red constituye una instancia participativa de intercambio de programas y noticias que facilitan más calidad de contenido y conocimiento del territorio.

A diferencia de la Comunidad Autónoma de Andalucía (que cuentan desde 1984 con la Asociación de Emisoras Municipales y Ciudadanas de Andalucía de Radio y Televisión, EMARTV), en la CARM no se ha constituido ninguna red que contribuya a facilitar la cohesión de las EM dentro del ámbito autonómico. Esta circunstancia, supone un déficit importante ya que los diferentes medios se encuentran en una situación de aislamiento que no propicia desarrollar acciones coordinadas en torno a intereses comunes: emisión de programación conjunta en determinadas franjas horarias (sobre todo, informativos de ámbito autonómico), captación de publicidad de grandes empresas regionales y nacionales, abordar la realización de proyectos de organismos e instituciones que precisan una cobertura supramunicipal o establecer una entidad de coordinación para defender intereses comunes.

En el panorama andaluz, los resultados son más satisfactorios gracias a la existencia de EMARTV. Del total de emisoras operativas actualmente (116), 62 emisoras (54\%) pertenecen a una red y 34 E.M. (29\%) se encuentran en un nivel óptimo de articulación territorial.

\subsection{Programación}

La programación como categoría que resume el resultado del trabajo conjunto es el capítulo más valorado. Analiza la emisión de informativos locales, de espacios con contenidos de proximidad, la variedad temática de los programas, la emisión de música no comercial, los programas con presencia de la radio en la calle y la realización de programas por parte de colectivos y colaboradores individuales.

La programación es el reflejo de todos los elementos que conforman la realidad comunicativa de la emisora: transparencia, pluralidad, participación social y especialización local de los contenidos. Además, suele estar condicionada por el carácter público del servicio y por la cantidad (variedad) y calidad (creatividad) de los recursos humanos y materiales disponibles. 
Entre los aspectos positivos de la programación podemos señalar el interés directo de los contenidos de proximidad, la posibilidad de la ciudadanía de ser protagonista y aportar información, y favorecer el conocimiento de la realidad local.

\section{Figura 4. Representación gráfica valores IRSCOM: categoría "Programación"}

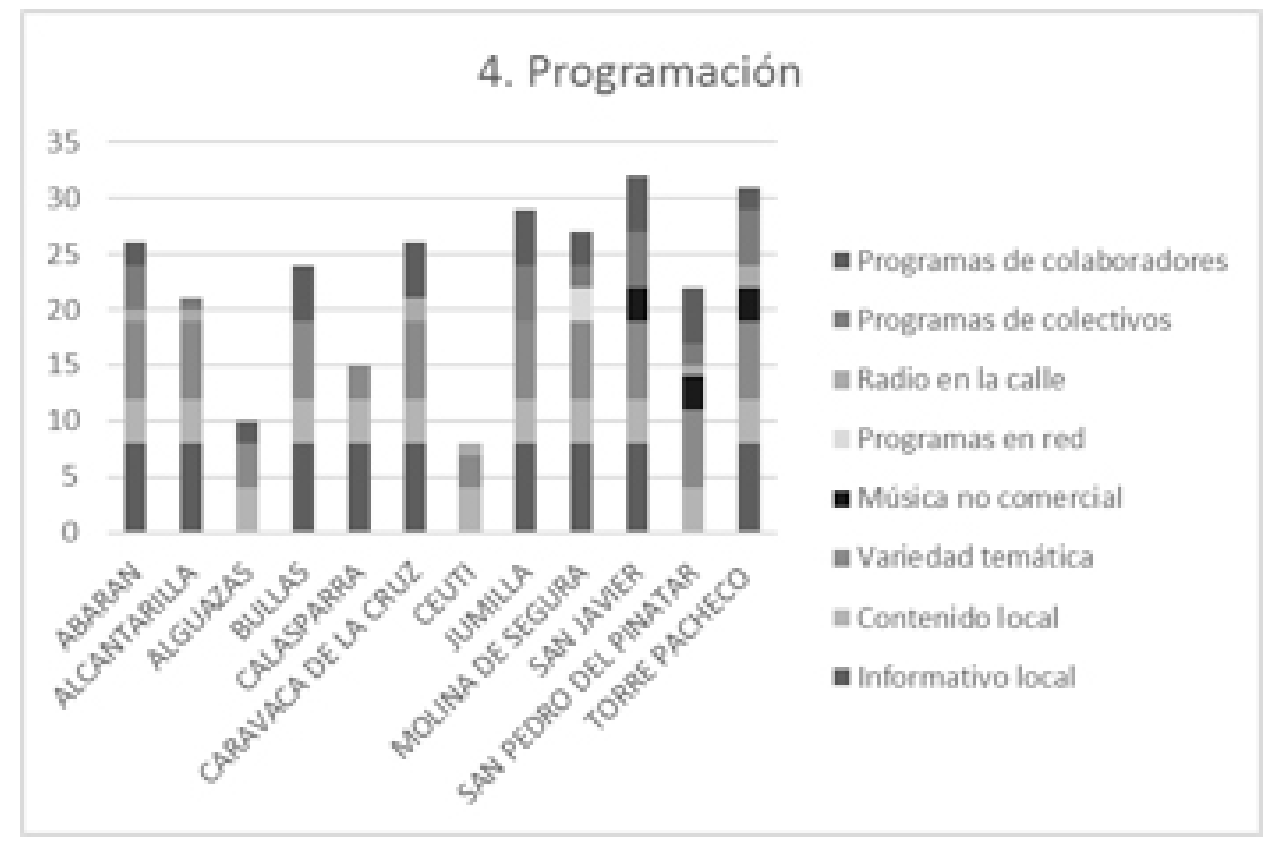

Fuente: elaboración propia (2017)

En la CARM, la mitad de las emisoras municipales de radio se sitúan en unos índices óptimos en lo que a programación se refiere. Del resto tres se encuentran muy cerca de cumplir los requisitos mínimos y las otras tres -que se corresponden con los municipios de menor poblacióntienen dificultades para organizar una parrilla comprometida, una debilidad que podría salvarse con la articulación en redes de intercambio (Figura 4).

Los resultados son muy similares a los registrados en la Comunidad Autónoma de Andalucía, donde 61 de las 112 EM evaluadas (54\%) se sitúan en valores óptimos, mientras que 51; un 46\%, no los alcanzan (Gabilondo, 2015).

Los altos índices de participación de los colectivos en la programación de las EM dan razón de ser a los medios de proximidad públicos. El alto número de programas realizados por la sociedad civil avalan la necesidad de apoyar a las EM en su apuesta por generar medios 
más horizontales e involucrados en la formación de imaginarios propios y, en definitiva, en la construcción democrática.

\subsection{Entorno comunicativo}

Esta categoría es considerada como la menos determinante en la configuración global del IRSCOM. Se examina la adaptación de la emisora al nuevo entorno comunicativo propiciado por la convergencia con internet y la telefonía móvil. Se trata de un esfuerzo adicional que deben realizar las EM para aprovechar las posibilidades del nuevo ecosistema comunicativo que viene siendo perfilado por la irrupción de la tecnología en los procesos de producción y emisión radiofónica, utilizando canales y soportes que propician un nuevo papel y experiencia de los usuarios.

En este apartado, se valora la actividad de la emisora a través de webs/blogs y en diferentes plataformas (Facebook, Twitter, Youtube, Linkedin, iVoox), la emisión (streaming), el acceso a contenidos online (podcast), la utilización de aplicaciones de mensajería instantánea (Whatsapp) y que la emisora cuente con una aplicación móvil.

Figura 5. Representación gráfica IRSCOM, categoría: “Entorno comunicativo"

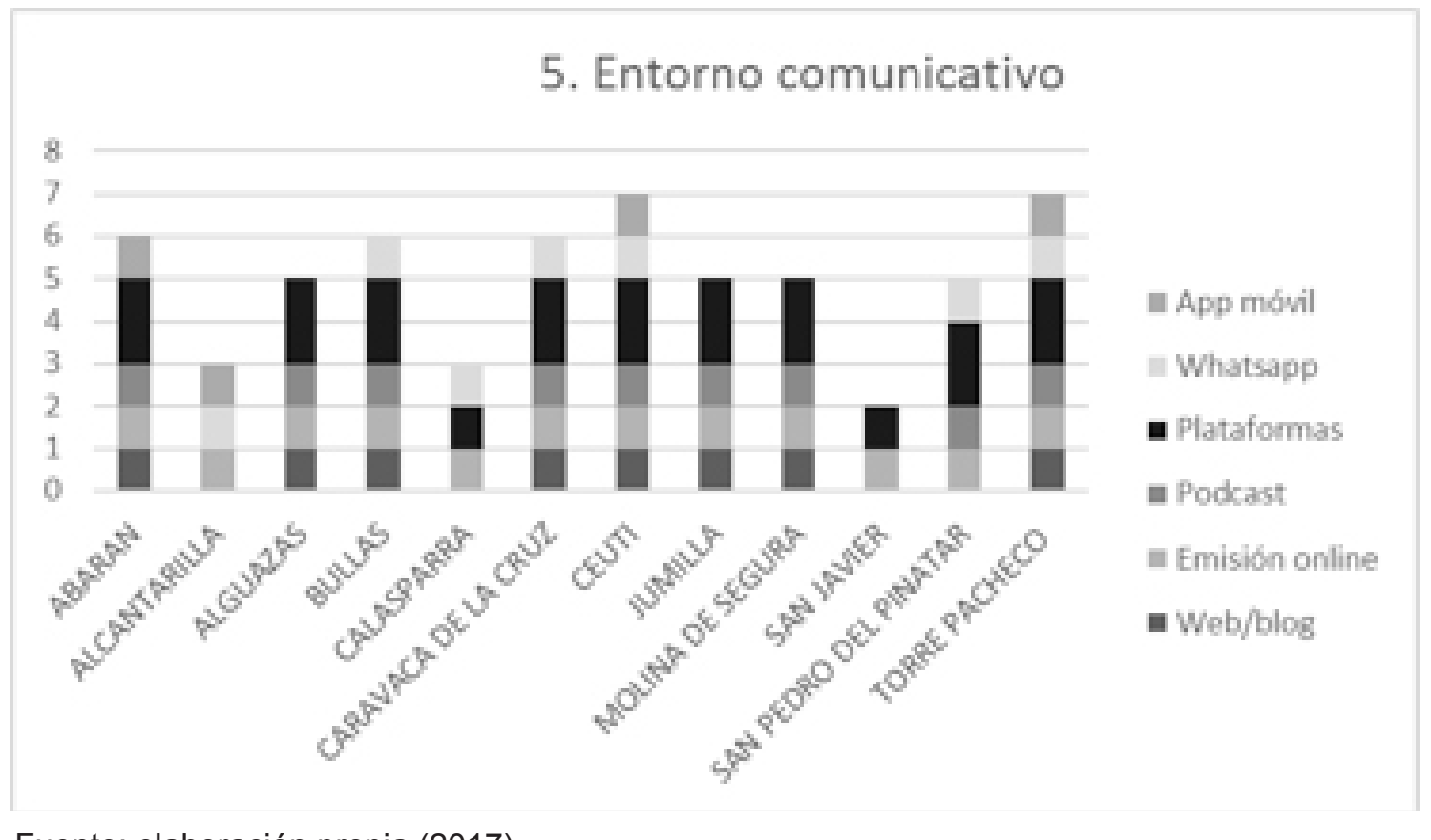

Fuente: elaboración propia (2017)

La mayoría de las EM murcianas (9 de 12, un 75\%) han desplegado un entorno comunicativo óptimo, mientras que las tres restantes; el 25\%, deben de realizar un esfuerzo adicional por implementar los nuevos servicios tecnológicos disponibles (Figura 5). 
En Andalucía, 69 de las 112 emisoras (62\%) se sitúan en un entorno comunicativo óptimo, mientras que 43 (38\%) muestran deficiencias (Gabilondo, 2015). Los datos murcianos superan ligeramente a los del panorama andaluz y demuestran el interés creciente de las EM en la configuración de un adecuado entorno comunicativo, utilizando las tecnologías y plataformas disponibles para desplegar todo su potencial relacional con los oyentes. Estas nuevas experiencias adecuan el medio a nuevos usos comunicativos (asincrónicos, ubicuos e interactivos), globalizar el alcance de las emisiones, implementar los canales de participación e interacción con la audiencia, conseguir interactuar con audiencias más jóvenes y tecnológicas, ofrecer experiencias de escuchas de contenidos a la carta e incorporar nuevos recursos expresivos y las nuevas narrativas de una realidad en transición.

\subsection{Infraestructuras}

El compromiso municipal con los medios de comunicación públicos debe procurar una dotación de dependencias y equipamiento, necesarios para la correcta realización del trabajo. En este sentido se valoran en este apartado los recursos técnicos disponibles, las instalaciones en general (estudios, sala de redacción y aula de formación) y la existencia de fondos sonoros y bibliográficos accesibles al público.

Todas las EM de la CARM menos la de la localidad de Calasparra (92\%), disponen de unas infraestructuras adecuadas para acometer el servicio con las garantías necesarias. Sin embargo, ninguna de ellas cuenta con una sala específica de formación y aquellas que realizan talleres de radio tienen que recurrir a otras dependencias municipales. Los fondos bibliográficos que poseen las emisoras son escasos o inexistentes y tampoco disponen de una fonoteca accesible al público. Es cierto que estos medios atesoran unos valiosos documentos sonoros sobre diferentes acontecimientos o protagonistas que pueden constituir la memoria histórica del municipio y que precisarían de un proceso de digitalización y catalogación para ponerlos a disposición de los vecinos. Por el contrario, los archivos fonográficos más recientes de estas emisoras se encuentran digitalizados y alojados en diferentes plataformas de distribución (iVoox, Zippyshare) o en la propia web de la emisora, lo que posibilita su conservación y el acceso abierto a los mismos en cualquier momento o desde cualquier lugar (Figura 6).

En Andalucía de las 112 emisoras analizadas, 65 (58\%) obtienen una valoración satisfactoria mientras que $47(42 \%)$ no cuentan con las instalaciones y los equipamientos suficientes (Gabilondo, 2015). 


\section{Figura 6. Representación gráfica valores IRSCOM: categoría "Infraestructuras"}

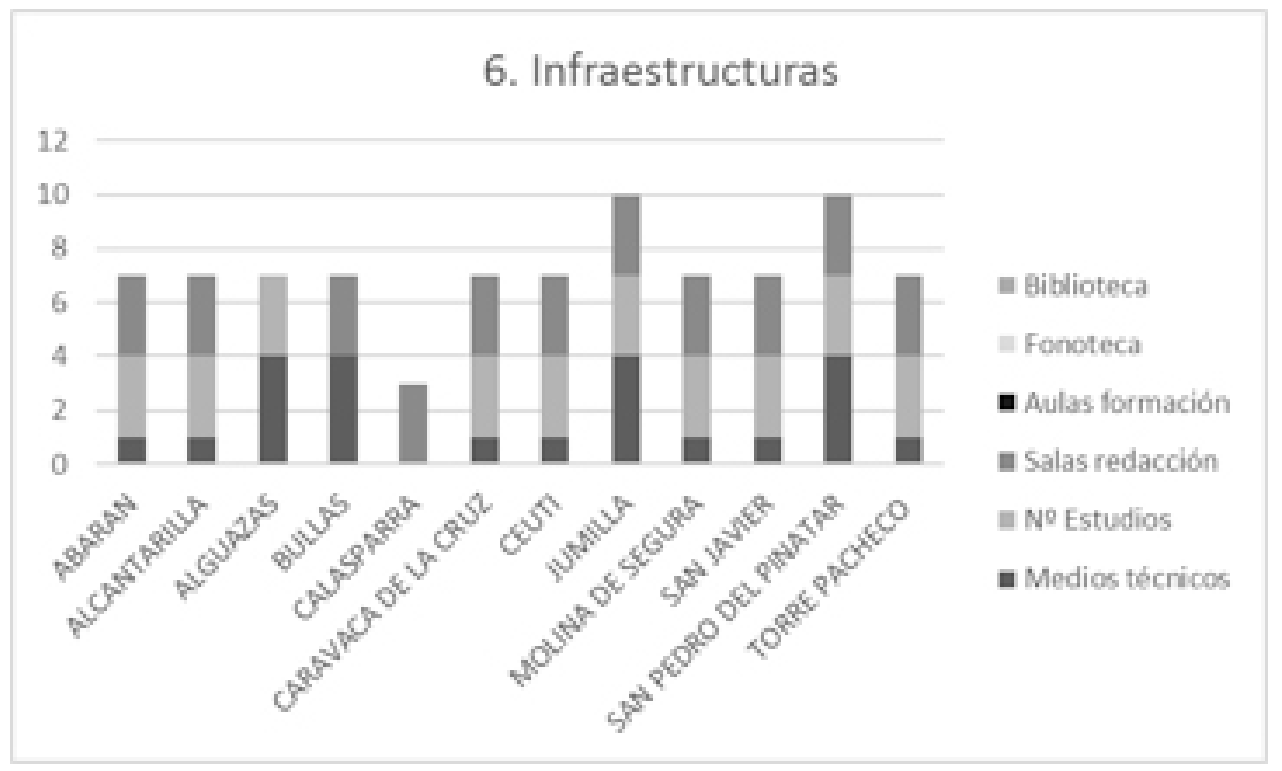

Fuente: elaboración propia (2017)

\section{RESULTADOS}

Esta investigación ha permitido diseñar el mapa de las emisoras municipales de radio de Murcia: 21 de los 45 ayuntamientos de la CARM poseen licencia para disponer de una emisora municipal de radio. Sin embargo, no todas las licencias se encuentran operativas ni se gestionan de acuerdo con lo establecido en la normativa específica: solo 12 de las 21 licencias (57\%) se encuentran operativas y ajustadas a la concesión otorgada, 3 (14\%) han privatizado su gestión, $5(24 \%)$ han tenido que cerrar y una licencia $(5 \%)$ no ha sido activada.

Andalucía, en 2013 , contaba con un $37 \%$ de emisoras operativas, un $11 \%$ habían sido externalizadas, el $30 \%$ se encontraban cerradas y el $22 \%$ de licencias no estaban operativas. En la CARM, más de la mitad de las licencias están operativas lo que pone de relieve un mayor compromiso de los ayuntamientos solicitantes por materializar y dar continuidad a sus concesiones, y gestionarlas de manera directa.

El proceso de externalización de la gestión y/o programación es similar en ambas comunidades, abriendo una brecha importante en el ADN de estos medios que necesitan de un mayor control en el cumplimiento de sus fines por las autoridades competentes en cada autonomía, tan responsables como los ayuntamientos en la continuidad de un delito de malversación y prevaricación. Los 
intereses mercantilistas propios de la gestión privada someten a las emisoras municipales externalizadas a criterios de gestión y programación que obedecen a la necesidad de conquistar audiencias masivas, debilitando la participación social y asentando criterios comerciales en la conformación de la programación.

Tabla 5. Ranking del IRSCOM de las emisoras municipales murcianas

\begin{tabular}{|c|l|c|c|c|}
\hline Puesto & \multicolumn{1}{|c|}{ Municipio } & Emisora & Población & Puntuación \\
\hline 1 & Torre Pacheco & Radio Torre Pacheco & 34.630 & 73 \\
\hline 2 & Molina de Segura & Radio Compañía & 69.614 & 68 \\
\hline 3 & Caravaca de la Cruz & Caravaca Radio & 25.591 & 66 \\
\hline 4 & Abarán & Radio Abarán & 13.183 & 63 \\
\hline 5 & San Pedro del Pinatar & Radio Pinatar & 24.660 & 62 \\
\hline 6 & Jumilla & Radio Jumilla & 25.362 & 61 \\
\hline 7 & San Javier & Radio San Javier & 31.782 & 57 \\
\hline 8 & Bullas & La Onda de Bullas & 11.714 & 55 \\
\hline 9 & Alcantarilla & Radio Sintonía & 41.155 & 46 \\
\hline 10 & Ceutí & Onda Color & 11.321 & 39 \\
\hline 11 & Calasparra & Radio Calasparra & 10.268 & 39 \\
\hline 12 & Alguazas & Alguazas Radio & 9.613 & 32 \\
\hline
\end{tabular}

Fuente: elaboración propia (2017)

Los valores obtenidos permiten establecer un ranking del IRSCOM de las emisoras municipales murcianas. Aunque las cifras de población no guardan una relación directa con la puntuación obtenida ya que el indicador pondera esta circunstancia con criterios de pertinencia, es cierto que en la realidad comunicativa estudiada, supone un determinante importante para un alto porcentaje de las mismas: cuatro de las emisoras que ocupan las últimas cinco posiciones (Alguazas, Calasparra, Ceutí y Bullas) se corresponden con aquellos municipios que tienen una población inferior a 12.000 habitantes: 2 de ellas cuentan con un solo trabajador y las otras 2 con dos trabajadores (Tabla 5). De ello solo se deriva una gestión que no ha sido capaz de dinamizar la apropiación del medio por las asociaciones y la ciudadanía en general.

En base a la baremación de las 6 categorías y las 33 áreas de análisis que componen el IRSCOM, la mitad de las emisoras municipales murcianas tienen una puntuación "muy baja" y la 
otra mitad una valoración "baja". Por este motivo, las emisoras tienen la necesidad de acometer aquellos proyectos necesarios para alcanzar un nivel de rentabilidad social superior que les sitúe entre los valores medios y óptimos. Los datos que arroja el presente análisis de la rentabilidad social de las emisoras municipales de radio murcianas pueden tomarse en consideración por parte de los gestores de estos medios, para conocer sus debilidades y fortalezas y a partir de ahí, incidir en aquellos aspectos que contribuyan a mejorar el servicio público que prestan a la población.

Los resultados ponen de manifiesto que las EM de radio murcianas destacan en programación, entorno comunicativo e infraestructuras; y presentan unos indicadores bajos en gestión, capital social y articulación territorial. En Andalucía, se alcanzan los mismos índices en lo que a programación se refiere, siendo notablemente superiores en capital social y articulación territorial; mientras que son ampliamente en gestión e infraestructuras. De acuerdo con el IRSCOM, podemos afirmar que tanto en Andalucía como en la CARM la mayoría de emisoras municipales desarrollan una óptima propuesta programática de acuerdo con parámetros de rentabilidad social. Las debilidades observadas en Murcia, en el capítulo de capital social y articulación territorial se compensa mínimamente con la gestión e infraestructuras. Al igual las debilidades andaluzas en gestión e infraestructuras penalizan las fortalezas en capital social y articulación territorial.

\section{CONCLUSIONES}

La utilización del espectro radioeléctrico por parte de actores privados no garantiza los contenidos de proximidad en las concesiones locales comerciales otorgadas, sobre todo en aquellas localidades de menor población donde la publicidad no deja gran margen de beneficios. La casi totalidad de estas emisoras comerciales se han convertido en postes repetidores de cadenas de radio que marginan la participación de actores locales. Esta realidad hace todavía más importante la defensa de los medios públicos y la evaluación de sus resultados mediante indicadores que permitan intervenir sobre las debilidades existentes. En este sentido, IRSCOM ha demostrado ser una herramienta eficaz. La réplica del indicador en un contexto diferente al que se ha desarrollado permite demostrar que su metodología es trasladable a otros ámbitos territoriales.

Es responsabilidad de las administraciones competentes garantizar el marco normativo adecuado y la dotación de recursos necesarios para que este servicio se preste en las mejores garantías de calidad y solvencia desde la transparencia, independencia, pluralidad y participación ciudadana.

La rentabilidad de los medios no puede evaluarse en términos económicos, su existencia se justifica por cuestiones de utilidad social. Por este motivo, la investigación se ha centrado en determinar la rentabilidad social de las EM con base en el Índice de Rentabilidad Social IRSCOM, 
diseñado por el Laboratorio de Comunicación y Cultura COMandalucía de la Universidad de Málaga. Los resultados muestran las fortalezas de estos medios: notable participación de ciudadanos y colectivos en la programación apuesta por la especialización local de los contenidos, variedad temática de la programación, óptima configuración de su entorno comunicativo mediante la utilización adecuada de las tecnologías y plataformas disponibles, y dotación de las dependencias y el equipamiento necesario. Al mismo tiempo, los datos señalan sus principales debilidades: falta de transparencia en la gestión, inexistencia de un consejo asesor municipal con participación ciudadana, dotación insuficiente de personal, e inexistencia de una red autonómica de emisoras municipales que propicie la realización conjunta y el intercambio de programas.

El indicador usado permite refrendar y concretar nuestra hipótesis de partida al comprobar que esta herramienta se muestra precisa para radiografiar las fortalezas y debilidades de los medios analizados y poder intervenir con medidas correctoras e incentivadoras.

El estudio realizado debe considerarse como una buena oportunidad para que los responsables autonómicos y municipales desarrollen políticas públicas conducentes a subsanar los problemas identificados.

Por ello, establecemos las siguientes propuestas de intervención:

- Adoptar un compromiso decidido por fomentar y consolidar estos medios, articulando medidas de apoyo que permitan su puesta en servicio en aquellos municipios en los que no se ha contemplado aún el proyecto, y garanticen su consolidación y viabilidad en aquellos otros que cuenten con emisora municipal de radio.

- Reconducir la situación de aquellas emisoras externalizadas o establecer su cierre por incumplimiento legal.

- Incentivar la calidad del servicio radiofónico público local dotando a estos medios de los recursos humanos y materiales necesarios desde la corresponsabilidad de las administraciones.

- Promover ayudas públicas para emisoras comprometidas en mejorar su IRSCOM.

- Fortalecer la construcción de asociaciones autonómicas para facilitar la articulación en red, el intercambio de programas y experiencias, y desarrollar iniciativas.

Dentro de la economía y sociedad globalizada en la que estamos inmersos se hace cada vez más necesario el desarrollo de políticas públicas que aborden la construcción cultural de la ciudadanía desde abajo, desde propuestas participativas horizontales que rescaten lo local y construyan identidad. En sentido, siguiendo las recomendaciones de la UNESCO, los indicadores se convierten en una pieza clave para fijar los comprotamientos éticos y la utilidad de los medios. 


\section{NOTAS}

${ }^{1}$ La actualización de resultados puede seguirse en www.com-andalucia.org [Fecha de acceso: 8 de agosto de 2018].

2 Puede consultarse en: https://com-andalucia.org/2017/05/20/el-laboratorio-de-comunicaciony-cultura-comandalucia-elabora-un-informe-para-ema-rtv-sobre-la-gestion-directa-de-losservicios-de-comunicacion-audiovisual/ [Fecha de acceso: 12 de noviembre de 2017].

${ }^{3}$ Con la finalidad de incluir otros servicios tecnológicos y de manera consensuada con el Laboratorio de Comunicación y Cultura COMandalucía de la Universidad de Málaga, en nuestro estudio hemos sustituido la denominación de "Presencia en internet" por la de "Entorno comunicativo", con el objetivo de incluir nuevas áreas de análisis como la utilización de Whatsapp y disponer de aplicación móvil.

\section{REFERENCIAS}

Chaparro, M. (1998). Radio pública local. Madrid: Fragua.

Chaparro, M. (2012). Medios de comunicación y democracia. Lecciones desde América Latina. Commons. Revista de Comunicación y Ciudadanía Digital, 1 (1), pp. 15-34.

Chaparro, M. (2015). Claves para repensar los medios y el mundo que habitamos. La distopía del desarrollo. Bogotá: Desde Abajo.

Chaparro, M.; Olmedo, S. y Gabilondo, V. (2016). El indicador de Rentabilidad Social en Comunicación (IRSCOM): medir para transformar. CIC. Cuadernos de Información y Comunicación, (21), pp. 47-62.

COMandalucía -Laboratorio de Comunicación y Cultura de la Universidad de Málaga- (2014). Disponible en: https://com-andalucia.org. Fecha de consulta: 8/11/2017.

Gabilondo, V. (2014). La construcción de territorio y ciudadanía por las radios públicas locales en Andalucía", en Chaparro, M. (Ed.) Medios de proximidad: participación social y políticas públicas. Málaga: iMedea/COMandalucía (pp. 163-182).

Gabilondo, V. (2015). Emisoras municipales en Andalucía. Proximidad y rentabilidad social (19792014) (tesis doctoral). Universidad de Málaga.

Laboratorio de Comunicación y Cultura COMandalucía de la Universidad de Málaga (2017). La gestión directa de los servicios de comunicación audiovisual de proximidad por los ayuntamientos. Recuperado de: https://com-andalucia.org/2017/05/20/el-laboratorio-de- 
comunicacion-y-cultura-comandalucia-elabora-un-informe-para-ema-rtv-sobre-la-gestiondirecta-de-los-servicios-de-comunicacion-audiovisual/. Fecha de consulta: 8/11/2017.

Olmedo, S. (2014). Irscom: la responsabilidad social de los medios y la construcción de indicadores democráticos, en Chaparro, M. (Ed.) Medios de proximidad: participación social y políticas públicas. Málaga: iMedea/COMandalucía (pp. 373-399).

Sánchez-Hernández, J.A. (2012). La radio pública local en la Región de Murcia. Murcia: ARTV.

\section{LEGISLACIÓN}

Ley Reguladora de las Bases del Régimen Local (Ley 7/1985, 2 de abril). Boletín Oficial del Estado, $n^{\circ}$ 80, 1985, 3 de abril.

Ley de Organización y Control de las Emisoras Municipales de Radiodifusión Sonora en Frecuencia Modulada (LOCEM) (Ley 11/1991, 8 de abril). Boletín Oficial del Estado, nº 85, 1991, 9 de abril (derogada).

Ley Orgánica 9/1992, de transferencia de competencias a Comunidades Autónomas que accedieron a la autonomía por la vía del artículo 143 de la Constitución (Ley Orgánica 9/1992, 23 de diciembre). Boletín Oficial del Estado, n 308, 1992, 24 de diciembre.

Ley General de Comunicación Audiovisual (Ley 7/2010, 31 de marzo). Boletín Oficial del Estado, $\mathrm{n}^{\circ} 79,2010,1$ de abril.

Ley de transparencia, acceso a la información pública y buen gobierno (Ley 19/2013, 9 de diciembre). Boletín Oficial del Estado, n² 295, 2013, 10 de diciembre.

Ley Audiovisual de Andalucía (Ley 10/2018, 9 de octubre). Boletín Oficial de la Junta de Andalucía, $n^{\circ} 200,2018,16$ de octubre. 